\title{
Digitale Museumsanwendungen
}

\author{
Digital Museum Appliances
}

Location Based Information_Museum Guide_Interactive Installations_Computer Graphics_Digital Storytelling

Zusammenfassung. Viele Museen suchen heutzutage neue Methoden und Wege, um Museen attraktiver zu gestalten und weitere Museumsbesucher und Zielgruppen anzusprechen. Eine Ausprägung davon stellen Science Center oder Themenparks dar, aber auch Kinder-, Kunst- und Technikmuseen mit digitalen Museum Guides oder „Exponaten zum Anfassen”. Basierend auf diesem Trend haben das ZGDV Darmstadt und die BauhausUniversität Weimar unabhängig voneinander multimediale Museumsanwendungen entwickelt, die insbesondere das jüngere Publikum in die Museen locken als auch „etablierte“ Museumsgänger durch einen digital erweiterten Wissens- und Erlebnisraum ansprechen soll. Die Fallbeispiele des ZGDV „DinoSim Senckenberg”, , art-E-fact " und das innovative Präsentationssystem „iPX" repräsentieren interaktive Museumsanwendungen zur wissenschaftlich-technischen Simulation von Dinosaurier Laufstudien und der Exploration von Gemälden und Kunstobjekten sowie des menschlichen Körpers innerhalb der seitens des Heinz Nixdorf Museumsforum initiierten Sonderausstełlung "Computer.Mensch" direkt vor Ort im Museum; "DinoExplorer Senckenberg" bietet eine virtuelle Umgebung zur spielerischen Exploration von Museumsräumen und Exponaten in Form einer emulierten Museumsrallye. Das digitale Osmantinum spricht den Literatur interessierten Besucher an und ist als mobiles Informations- und Führungssystem auf der Basis eines PDA's konzipiert, ohne die besondere Stimmung der Museumsräume zu stören.
Summary. Many museums nowadays search for new methods to make the museum more attractive and to reach new audiences. Based on this trend the ZGDV Darmstadt and the Bauhaus-University Weimar independently developed multimedia applications that address young audiences as well as "traditional" visitors and offer access to digitally enhanced Knowledge- and Experience Spaces.

The works of ZGDV "DinoSim Senckenberg", ,art-E-fact" and an innovative presentation system „iPX" represent interactive applications for the museuph for the scientific-technical simulation of dinosaurs movement, the exploration of paintings and art-objects and the human body in the Heinz Nixdorf Museum. „DinoExplorer Senckenberg" offers a virtual environment for the playful exploration (museum rally) of spaces and objects in the museum. The "Digital Osmantinum" addresses visitors interested in literature and is realized as a PDA based, mobile information and guidance system that does not interfere with the special aura of the museums rooms.

\section{DinoHunter Senckenberg}

Im Rahmen der Neueröffnung des Senckenberg Naturkundemuseums im November 2003 sollte das Ausstellungsangebot durch die Integration von Multimedia-Anwendungen angereichert werden. Hierfür hat die Storytelling Abteilung des ZGDV Darmstadt innerhalb des Projektes "DinoHunter Senckenberg" in Kooperation mit T-Online und ion2s -

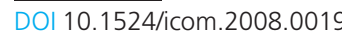

buero fuer Interaktion die Terminal Anwendung "DinoSim Senckenberg" und die 3D Anwendung „DinoExplorer Senckenberg" konzipiert und implementiert. Die Grundidee des DinoHunter Gesamtkonzeptes besteht hierbei in der Verknüpfung von traditionellen Medien und Exponaten in Museen mit "hightech" und "Neuen Medien", z.B. der Computergraphik, des Interactive Digital Storytelling und des User Centered Design.

Hierbei wird - im Gegensatz zu verschiedenen Film- und TV Produktionen - besonderer Wert auf eine möglichst detaillierte und natürliche Darstellung der Dinosaurier (Aussehen, Farbe, Häute; Laufstil) gelegt, die auf aktuellen Forschungsergebnissen der Paläontologen des Senckenberg Museums beruhen.

\subsection{DinoExplorer Senckenberg}

DinoExplorer Senckenberg, realisiert als Suchspiel, bietet die Möglichkeit das Senckenberg Museum am PC zuhause virtuell zu besuchen und sich vorab zu informieren oder nach einem Museumsbesuch bestimmte Themengebiete zu 
vertiefen und auf spielerische Art und Weise Zusatzinformationen über bestimmte Ausstellungsobjekte zu erhalten.

Mit Hilfe eines elektronischen Assistenten in Form eines emulierten PDA begeben sich die Anwender in einer virtuellen Umgebung (vereinfachtes 3D Modell des Senckenberg Museums) auf die Suche nach einem speziellen Exponat/Urgestein (Leptictidium), das sich im Untergeschoß des Museums im "Grube Messel Raum" befindet. Sobald das Leptictidium entdeckt wurde, wird auf dem emulierten PDA neben den allgemeinen Informationen und Hinweisen zur Belohnung eine Zusatzfunktionalität frei geschaltet: So erwachen die ausgestellten Dinosaurierskelette zum Leben und man kann diese anschließend mit verschiedenen Häuten und Haaren betrachten und die Dinos auch atmen sehen.

\subsection{DinoSim Senckenberg}

DinoSim Senckenberg ist als Terminalanwendung vor Ort im Museum realisiert und zeigt eine interaktive Laufstudie der beiden bedeutendsten Dinosaurier im Senckenbergmuseum: Tryrannosaurus Rex (T-Rex) und der Schlangenhals-Dinosaurier Diplodocus. Besucher können auf Terminals direkt vor den Skeletten das jeweilige Tier in voller Bewegung von allen Seiten aus betrachten, sich via Touchscreen bedrohlich



Bild 1: DinoSim Senckenberg - Skelette und Multimedia

nahe an die Dinos hinbewegen oder die Dinos in ihrem schönsten Glanze erscheinen lassen. Zusätzlich können individuelle Screenshots mit dem eigenen Blick auf die Dinos geschossen werden; diese Bilder werden dann per Email nach Hause geschickt oder unter Nutzung des T-Online Fotoservices auf Taschen, T-Shirts oder Tassen gedruckt.

DinoSim Senckenberg ist seit 2003 bis heute Tag für Tag im Einsatz und erfreut sich höchster Beliebtheit; nicht nur bei Kindern, sondern auch bei allen anderen Besuchern, die wissen möchten wie Dinos vielleicht mal ausgesehen haben können oder wie sich diese fortbewegt haben. Evaluationsstudien haben einerseits den völlig unbefangenen und gewohnten Umgang der Kinder und Jugendlichen ( $\rightarrow$ digital natives generation) mit derartigen neuen Medien und Tech- nologien gezeigt, andererseits aber auch die abnehmende Scheu der älteren Generation und die Potentiale derartiger Medien für Lehr- und Lernzwecke.

\section{Mixed Reality Platt- form art-E-fact}

Ähnliche Erkenntnisse hat die Storytelling Gruppe des ZGDV innerhalb zahlreicher Usability Studien im Kontext des von der EU geförderten Projektes art-E-fact gesammelt: Zur interaktiven Wissensvermittlung von Kunstobjekten/Gemälden wurde eine Mixed Reality Plattform entwickelt, die es einerseits Autoren und Künstlern erlaubt, interaktive Museumsinstallationen zu konzipieren und Inhalte in interaktive Geschichten zu verpacken; andererseits können die Anwender im Museum so auf ganz natürliche Art und Weise Gemälde explorieren oder in Form von Geschichten und Dialogen zwischen virtuellen Charakteren, die z.B. die Rolle eines Kunsthistorikers oder Kunstanalytikers vertreten, etwas über die Hintergründe von Exponaten und deren Künstler erfahren.

Insbesondere die natürliche Exploration von Gemälden mittels Zeigegesten unter Nutzung des innovativen Präsentationssystems „ „PX" fand in den art-E-fact Ausstellungen in San Sebastian, Florenz und Wien großen Anklang: Besucher al-

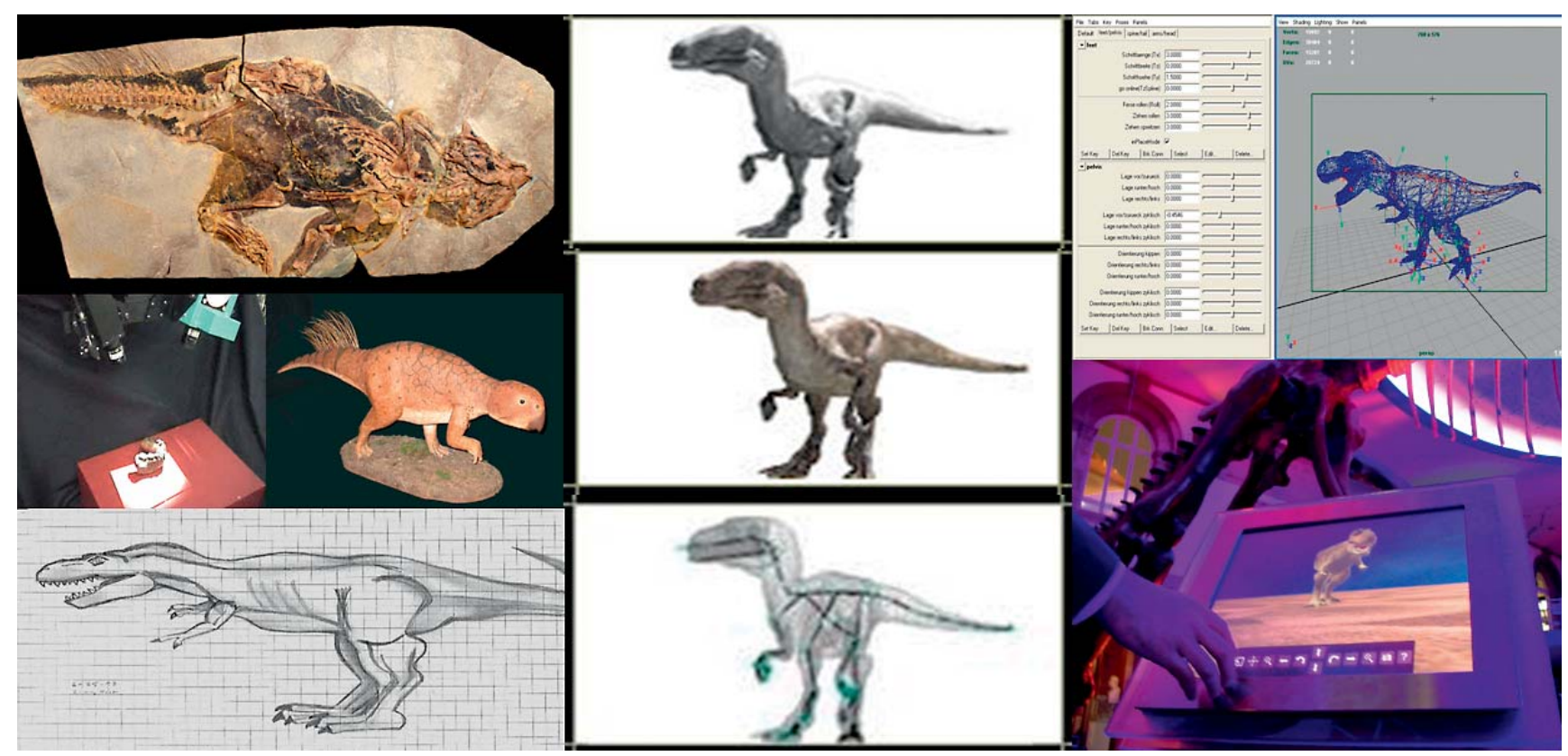

Bild 2: Entwicklungsprozess von DinoSim Senckenberg - von Fossilienfunden über 3D Rekonstruktionen, Extraktion von Geometrie, Skelett und Aussehen, Modellierungs- und Animationssoftware (Maya) bis hin zur Touchscreen Applikation auf Terminals 

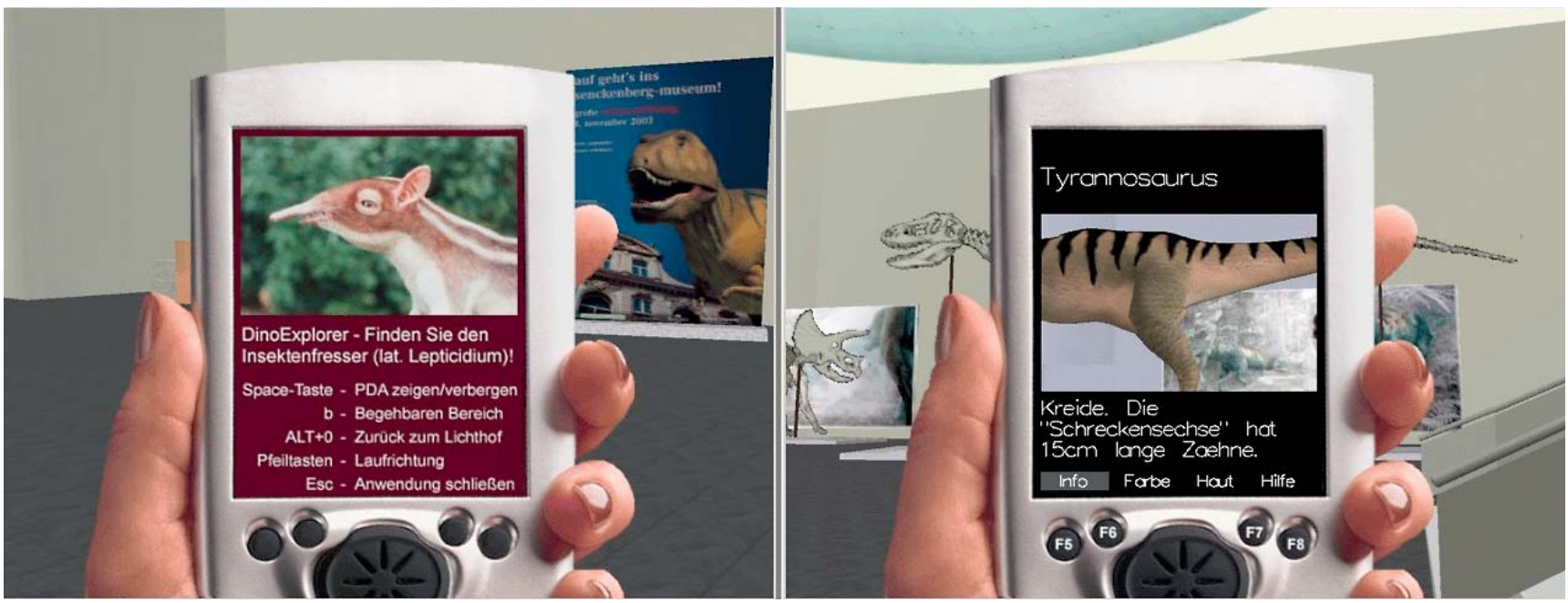

Bild 3: DinoExplorer Senckenberg

ler Altersschichten und Besuchergruppen waren von der natürlichen Interaktionsform und den durch den Einsatz von physischen Devices (Lupe, Taschenlampe, Schwamm) und Computer Vision Technologien (Bilderkennung, Tracking) hervorgerufenen visuellen Effekten sehr beeindruckt.

So können die Besucher mit Lupe und Taschenlampe einzelne Bereiche eines Gemäldes vergrößern und hervorheben oder mit dem Schwamm im Falle von übermalten Gemälden darunter liegende Schichten frei legen. Auch die Story-basierte Vermittlung von Inhalten und Anekdoten rund um spezifische Exponate wurde positiv wahrgenommen; jedoch besteht hierbei die Schwierigkeit, dass die Besucher im allgemeinen - vor allem bei Führungen - nur eine begrenzte Zeit an einzelnen Exponaten verweilen; die Geschichten und Dialogsequenzen je- doch eine gewisse Zeit in Anspruch nehmen.

\section{Präsentations- system iPX}

Die iPX Technologie wurde als Leitexponat des "Anatomischen Theaters" innerhalb der seitens des Heinz Nixdorf Museumsforum initiierten Sonderausstellung "Computer.Medizin" (Oktober 2006 - Mai 2007) erfolgreich eingesetzt und ist nun Teil der gleichnamigen Wanderausstellung (seit September 2007 in Dortmund). Durch Zeigegesten - ohne zusätzliche Hilfsmittel - können die Besucher mit digitalen Welten interagieren und Haut, Nerven- und Kreislaufsystem sowie das Skelett des menschlichen Körpers auf einem Großbildschirm betrachten.
¡PX verwendet für die technische Realisierung ausschließlich Standard-Hardware-Komponenten: Ein handelsüblicher, leistungsstarker PC und zwei Fire-Wire Kameras (IEEE 1394) reichen aus, um sowohl die video-basierte Erkennung der Zeigegestik als auch die Darstellung der interaktiven, virtuellen Welten auf dem Ausgabegerät (z.B. Flachbildschirm, Holodisplay oder große Projektionssysteme wie eine PowerWall) in Echtzeit zu berechnen. Die strikte Trennung der Eingabe- und Ausgabesoftwaresysteme als eigenständige Anwendungen macht die Generierung neuer Inhalte und das Pflegen bereits existierender Daten besonders einfach: Für das Rendering wird u.a. das ZGDV eigene System Avalon eingesetzt, das den kompletten Umfang und die Funktionalität von Standards wie VRML und X3D zur Verfügung stellt und so die Interaktion sowohl mit zweidimen-

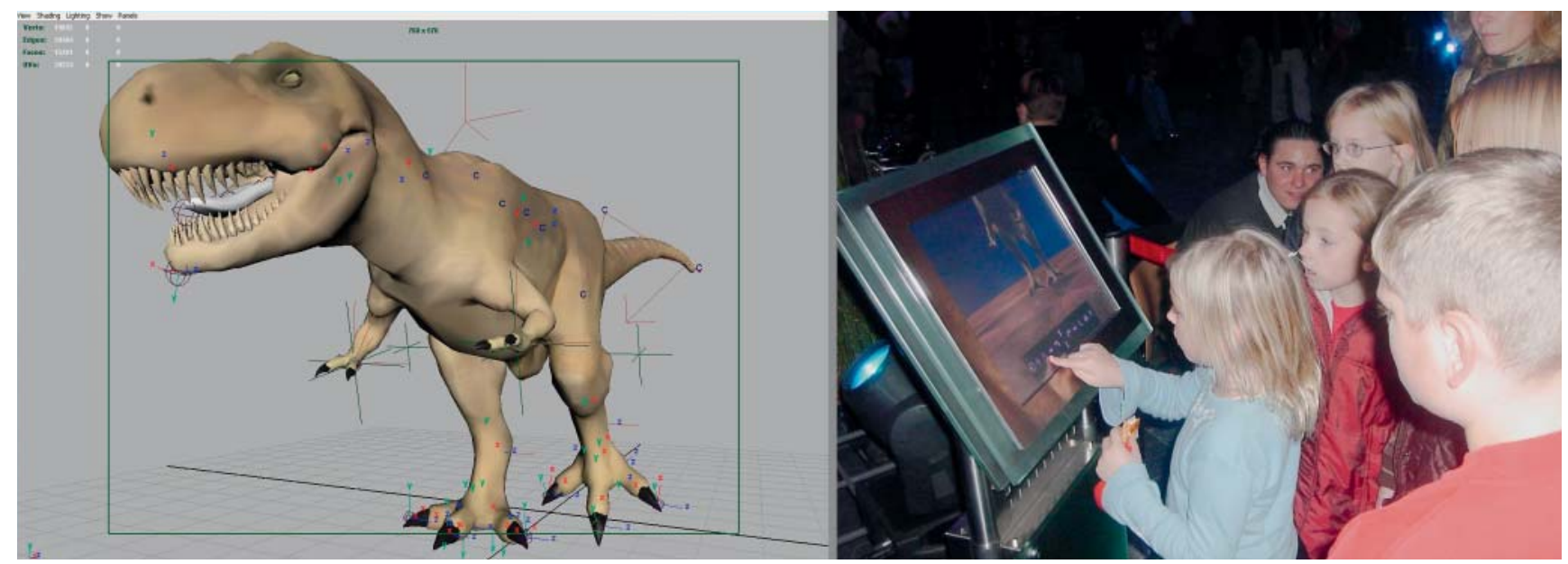

Bild 4: DinoSim Senckenberg - Dino Laufstudien des T-Rex und Diplodocus 


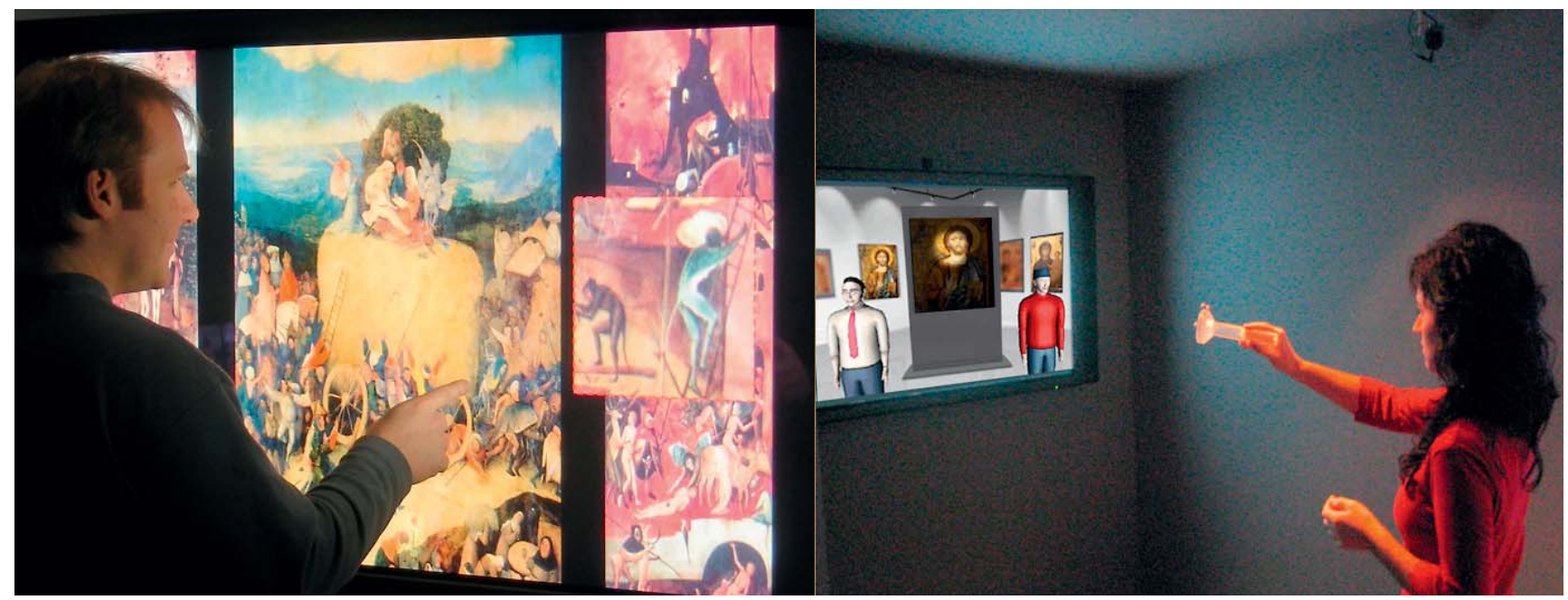

Bild 5: art-E-fact - Mixed Reality Plattform zur interaktiven Exploration von Kunst
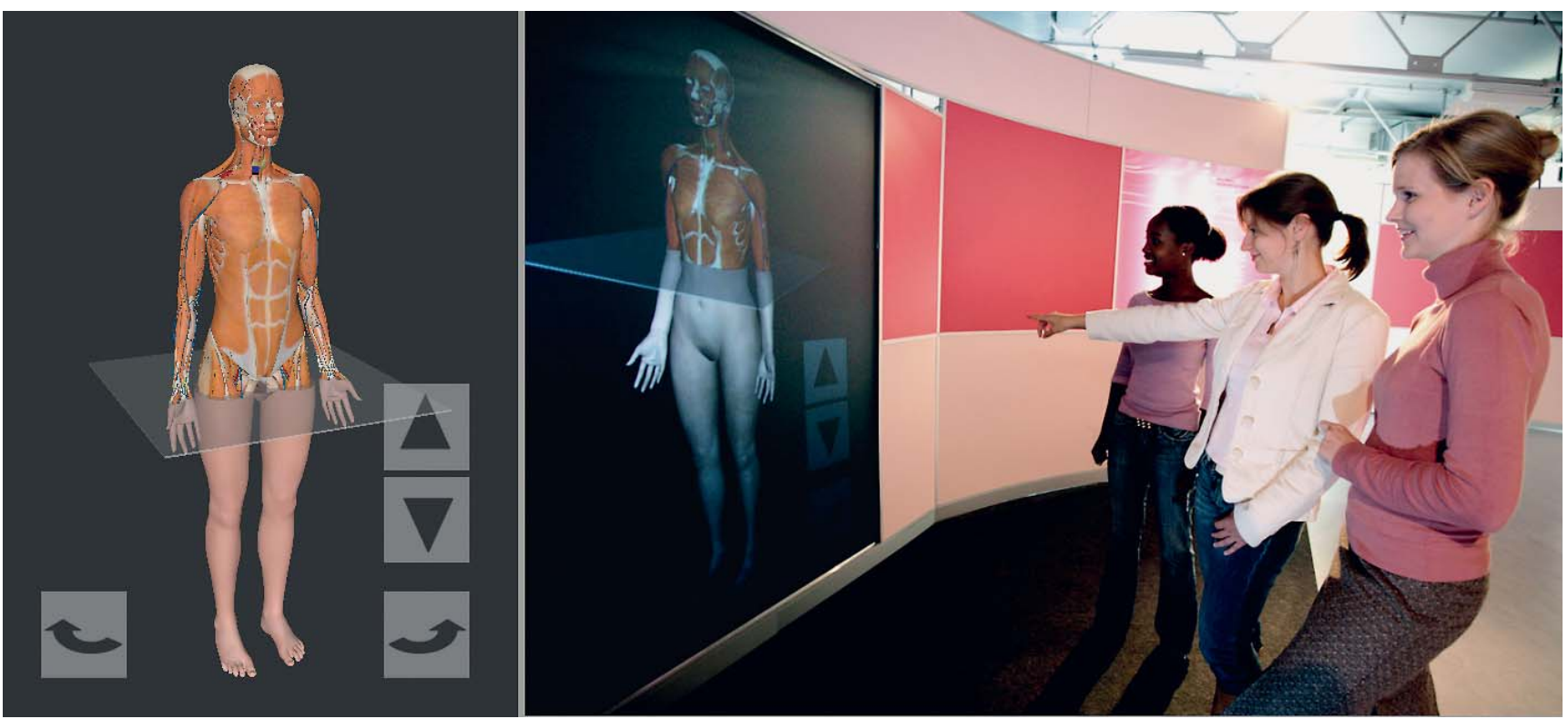

Bild 6: iPX Präsentationssystem in der Mensch.Medizin Ausstellung

sionalen Bildern wie auch die Darstellung und Manipulation von dreidimensionalen Objekten oder die Steuerung von Standard-Anwendungen (z.B. Webseiten, Flash oder Powerpoint) ermöglicht.

\section{Das digitale Osmanti- num - ein ortsbasiertes Museumsführungssystem}

Im Bereich der mobilen Museumsanwendungen wurde von der Bauhausuniversität Weimar in Zusammenarbeit mit dem Betreiber des Wieland-Museums Ossmannstedt, der Klassikstiftung Weimar ein mobiles PDA System entwickelt, das den Einsatz anderer Medien im Ausstellungsraum überflüssig machen soll, um die Atmosphäre in den Museumsräumen, den ursprünglichen Wohn- und Arbeitsräumen Christoph Martin Wielands, nicht zu stören. Gleichzeitig sollte aber das umfangreiche Wirkungsgebiet und die Arbeit Wielands mit medialen Mitteln sichtbar gemacht werden.

Unser Ansatz ist daher ein mobiles Informations- und Führungssystem auf der Basis eines PDA's, das sich auditiver und visueller Kanäle bedient. Der PDA selbst wurde in einer Hülle verpackt, die nur den Touch-Screen selbst sichtbar lässt. Die Form der Verpackung orientiert sich dabei an einem Buch. Das Gerät kann bequem umgehängt und in das Museum mitgenommen werden.

Um den Besuchern eine möglichst einfache Orientierung in den Räumen und

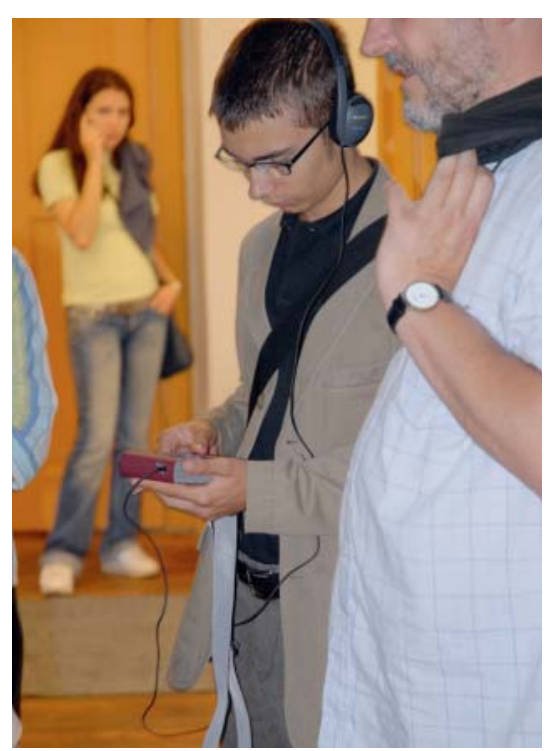

Bild 7: PDA-System mit Kopfhörer 
auch in dem umfangreichen multimedialen Informationssystem anzubieten, wurden drei verschiedene Interaktionsprinzipien angewandt:

- Halbautomatische Führung durch Bewegung im Museum - WLAN-Positioning

- Stift basiertes Auswählen einzelner Inhalte auf dem Touch Screen

- Gesten basiertes Sammeln von Informationen - RFID-Labels

Die halbautomatische Führung bietet die Möglichkeit einen Überblick der verschiedenen Arbeitsgebiete Wielands zu erlangen, während das Museum durchschritten wird. Dabei wird der PDA durch ein WLAN-Positioning System lokalisiert und eine einführende Information akustisch und visuell zu den jeweiligen Räumen gegeben. Diese Form der Führung richtet sich an Besucher, die möglichst schnell und einfach einen Überblick zu Leben und Werk von Wieland erhalten wollen.

Um weitere Informationen zu einzelnen Ausstellungsobjekten, Themengebieten, Personen, etc. zu erhalten, verwendet der Besucher die Screenbasierte Interaktion per Stift oder Finger und wählt die entsprechenden Objekte auf der PDA-Oberfläche aus.

Da die Lokalisierungssoftware nur eine Genauigkeit von etwa 2 qm bietet, entschieden wir uns dazu, einzelne Orte (im vorliegenden Fall einzelne Büsten) durch RFID-Labels zu markieren und dem Besucher das Sammeln dieser Informationen zu erlauben. Hierzu wurde der PDA mit einem RFID Reader ausgestattet, der in einer Entfernung von ca. 15-30 cm zu den entsprechenden Labels eine ID ausliest und den korrespondierenden Inhalt auf dem PDA darstellt.

Alle Inhalte werden entweder via WLAN von einem zentralen Server geladen oder von einer integrierten MMC Karte gelesen. Die Datenbank auf dem Server ist erweiterbar und so können neue Inhalte oder sogar neue Führungen bequem eingespeist werden, ohne umfangreiche Aktualisierungen an den Ausleihgeräten vorzunehmen.

Weitere Systeme, die sich der Lokalisierung durch GPS gestützte Daten bedienen, wurden ebenfalls bereits von uns implementiert und untersucht. ZooGo (http://www.lossebon.de/zoogo/_zoogo.



Bild 8: Screen Bibliothek

html), Annas Geheimnis (http://annasgeheimnis.de).

Zukünftige Arbeiten sollen sich weiter mit der Verbindung von verschiedenen Lokalisierungsmethoden (Außenraum Innenraum) und dem Zugang zu ortsbasierten Informationen beschäftigen. Unbedingt sollen dabei auch neue Interaktionsformen untersucht werden und Benutzer generierte Inhalte einbezogen werden.

\section{Zusammenfassung}

Der Einsatz von Neuen Medien und digitalen, multimedialen Museumsanwendungen bietet eine Vielzahl neuer Möglichkeiten der Informationsvermittlung.

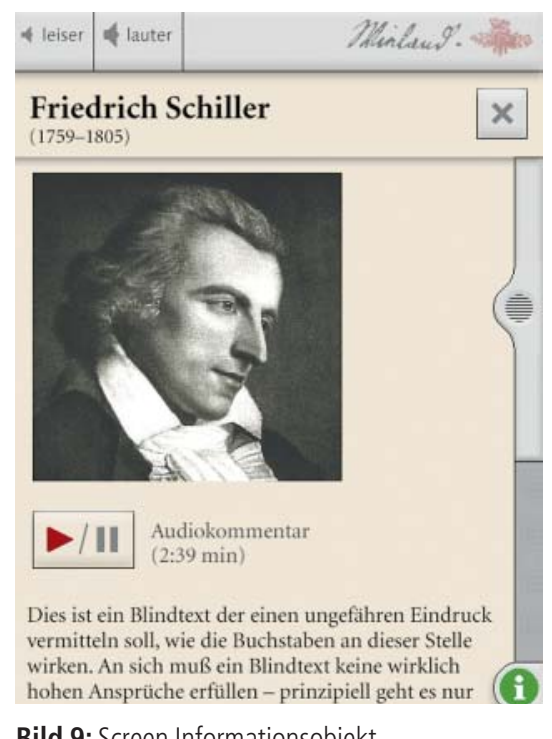

In Archiven verborgene Schätze können durch digitale Technologien sichtbar gemacht werden. Die Vor- und Nachbereitung des Museumsbesuchs, der personalisierte Zugang zu Informationen, das Erlebnis des Museumsbesuchs selbst, und viele weitere Beispiele sind noch weitgehend technologisch unerschlossene Arbeitsfelder und machen den Besuch unserer Museen attraktiver und erschließen neue Zielgruppen. Neben den wirtschaftlichen und organisatorischen Aspekten ist auch ein stabiler und ökonomischer Betrieb von Bedeutung. Einige Beispiele wurden vorgestellt.

Zur Erstellung und Steuerung der in diesem Beitrag skizzierten Museumsanwendungen hat das ZGDV Darmstadt eine umfassende Storytelling Plattform samt Autorenwerkzeug, Story Engine und verschiedenen Rendering-Systemen konzipiert und realisiert. Innerhalb des von der EU im 7. Rahmenprogramm im Bereich „Technology-enhanced Learning" geförderten Projektes 80Days wird diese Plattform weiterentwickelt und die Symbiose von E-Learning, Storytelling und Gaming Konzepten in mobilen Edutainment Anwendungen erforscht.

\section{Literatur}

Geelhaar, J.: Interaktion mit verteilten digitalen Informationsräumen, pp. 225-236. In: (Eibl, M.; Reiterer, H.; Stephan, P.F.; Thissen, F.; Eds.): Knowledge Media Design - Theorie, Methodik, Praxis. 2. Aufl., Oldenbourg München, 2006.

Geelhaar, J. et al.: An Example for Location Sensitive Media Integration: Re-discovering the Place Itself as a Medium by Adding Technology, pp. 270-276. In: (Göbel, S. et al.; Ed.): Technologies for Interactive Digital Storytelling and Entertainment. TIDSE 2004, LNCS 3105, Springer Berlin/Heidelberg, 2004

Geelhaar, J.: Technische Strategien der Interaktion in verteilten Informationsräumen. pp. 53-62. In: HyperWerk FHBB (Ed.): rtrp - right time right place, Verlag HyperWerk FHBB Basel, 2004.

Göbel, S.; Konrad, R.; Salvatore, L.; Sauer, S.; Osswald, K.: U-CREATE: Authoring Tool for the Creation of interactive Storytelling based Edutainment Applications. In: (Cappellini, V.; Hemsley, F.; Eds.) Electronic Imaging \& the Visual Arts. Proceedings EVA 2007 Florence, pp. 53-58. Pitagora Editrice Bologna.

Göbel, S.; Feix, A.: MuViPlan: Interactive Authoring Environment To Plan Individual Museum Visits. In: (Trant, J.; Bearman, D.; Eds.) Museums and the Web 2005. Proceedings (CDROM: ISBN 1-885626-31-2) Toronto: Archives \& Museum Informatics, March 31, 2005. 
Hoffmann, A.; Göbel, S.; Schneider, O.; lurgel, I.: Storytelling Based Edutainment Applications. In: (Tan, L.; Subramaniam, R.; Eds.) E-Learning and Virtual Science Centers (ISBN 1-59140-592-0), pp 190-214, Information Science Publishing, Hershey, 2005.

Malerczyk, C.: Dynamic Gestural Interaction with Immersive Environments. In: (Cunninghan, S.; Ed.): European Association for Computer Graphics (Eurographics): Proceedings of WSCG 2008. Communication Papers.

Malerczyk, C.; Dähne, P.; Schnaider, M.: Exploring Digitized Artworks by Pointing Posture Recognition. 6th International Symposium on Virtual Reality, Archaeology and Cultural Heritage. Pisa, Italy, 8th-11st November 2005

Sauer, S.; Osswald, K.; Göbel, S.; Feix, A.; Zumack, R.: Edutainment Environments. A Field Report on DinoHunter: Technologies, Methods and Evaluation results. In: (Bearman, D.; Trant, J.; Eds.) Museums and the
Web 2004: Selected Papers from an International Conference. Toronto: Archives \& Museum Informatics. 165-172.

Sauer, S.; Göbel, S.: Focus your young visitors: - Kids Innovation - Fundamental changes in digital edutainment. In: (Bearman, D.; Trant, J.; Eds.) Museums and the Web 2003: Selected Papers from an International Conference. Toronto: Archives \& Museum Informatics. 131-141.
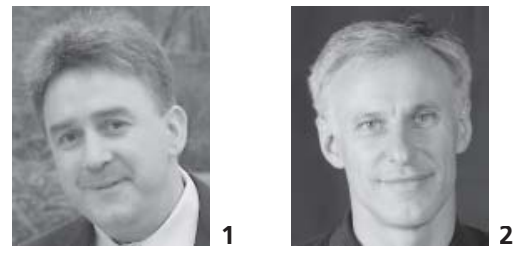

1 Dr.-Ing. Stefan Göbel leitet die Storytelling Gruppe am ZGDV Darmstadt und ist Geschäfts- führer des InnoGames Forum. Seine Forschungsschwerpunkte liegen im Bereich Storytelling basierte Edutainment Anwendungen und Serious Games. Dr. Göbel ist Initiator der internationalen Storytelling Konferenz "TIDSE" als auch der Science meets Business Veranstaltung "GameDays", die jährlich in Darmstadt stattfinden.

E-Mail: stefan.goebel@zgdv.de

www.zgdv.de, www.innogames-forum.de

2 Jens Geelhaar, Prof. Dr.sc.hum., Dipl.chem., Dipl.Kunst, ist Professor für Interface Design an der Bauhaus-Universität Weimar. Die Schwerpunkte der Professur liegen in der Konzeption und Gestaltung von interaktiven Schnittstellen in Screen basierten Anwendungen sowie mobilen und ubiquitären Medien in architektonischen und urbanen Räumen. Prof. Geelhaar ist stellvertretender Sprecher des Arbeitskreises "Knowledge Media Design" der GI.

E-Mail: jens.geelhaar@medien.uni-weimar.de

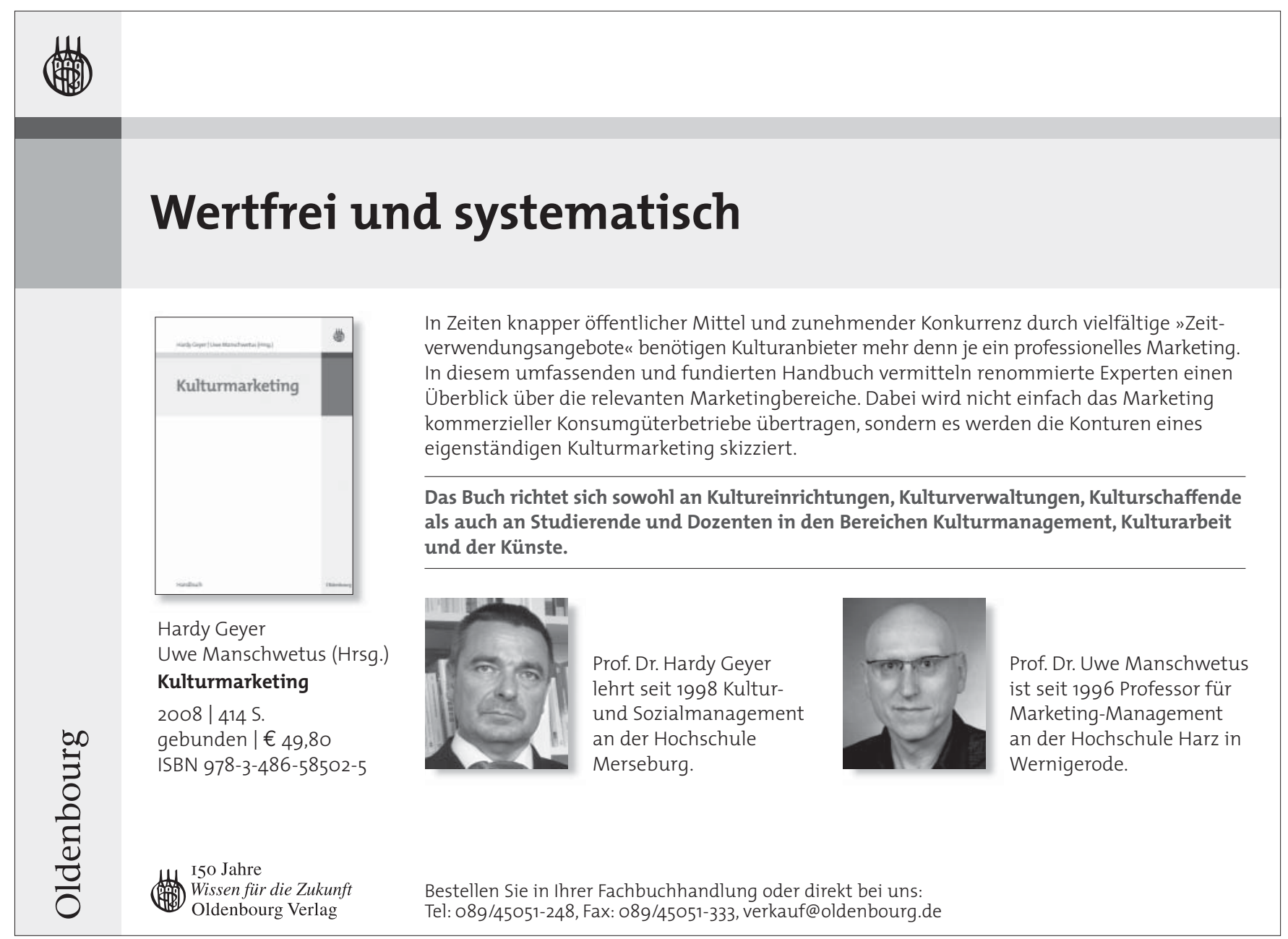

